\title{
Universal spatio-topological control of crystallization in sessile droplets using non- intrusive vapor mediation
}

Cite as: Phys. Fluids 33, 012101 (2021); https://doi.org/10.1063/5.0037120

Submitted: 11 November 2020. Accepted: 08 December 2020 . Published Online: 06 January 2021

Omkar Hegde, Ankur Chattopadhyay, and Saptarshi Basu

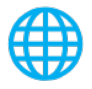

\section{ARTICLES YOU MAY BE INTERESTED IN}

Analyzing the dominant SARS-CoV-2 transmission routes toward an ab initio disease spread model

Physics of Fluids 32, 123306 (2020); https://doi.org/10.1063/5.0034032

Insights on drying and precipitation dynamics of respiratory droplets from the perspective of COVID-19

Physics of Fluids 32, 123317 (2020); https://doi.org/10.1063/5.0037360

Numerical analysis of combined electroosmotic-pressure driven flow of a viscoelastic fluid over high zeta potential modulated surfaces

Physics of Fluids 33, 012001 (2021); https://doi.org/10.1063/5.0033088

\section{Physics of Fluids GALLERY OF GOVERS}




\title{
Universal spatio-topological control of crystallization in sessile droplets using non-intrusive vapor mediation
}

\author{
Cite as: Phys. Fluids 33, 012101 (2021); doi: 10.1063/5.0037120 \\ Submitted: 11 November 2020 - Accepted: 8 December 2020 • \\ Published Online: 6 January 2021
}

\author{
Omkar Hegde, (D) Ankur Chattopadhyay, and Saptarshi Basu
}

AFFILIATIONS

Department of Mechanical Engineering, Indian Institute of Science, Bangalore 560012, India

a) Author to whom correspondence should be addressed: sbasu@iisc.ac.in

\begin{abstract}
The present work showcases a mechanism of asymmetric solvent depletion using vapor-mediated interaction that can non-intrusively regulate the site of crystal precipitation. In general, the flow pattern inside a drying sessile saline droplet leads to circumferential deposition of salt crystals at the end of evaporation. Instead, we show that our proposed approach can manipulate the spatial location of crystal precipitation. The introduction of a pendant ethanol droplet near the sessile saline droplet's vicinity creates an asymmetric ethanol vapor gradient around the sessile drop. The differential adsorption of ethanol vapor on the surface of the saline droplet gives rise to a spatial surface tension gradient. This, in turn, enhances the internal convection $\left[\sim \mathrm{O}\left(10^{2}-10^{3}\right)\right]$ within the saline droplet. The vigorous and non-uniform flow promotes targeted contact line depinning, ensuring preferential segregation of the salt crystals. Using this methodology, we can inhibit crystal formation at selected locations and favorably control its deposition in definite regions. The interplay of flow hydrodynamics and the associated contact line motion governs this phenomenon marked by the inception and growth of crystals at a preferential site. The universal character of such a phenomenon is verified for a variety of salt solutions on the glass substrate. Tweaking of contact line motion using vapor mediation can be used as a strategic tool for controlling the salt crystallization sites for various potential applications starting from water purification to portable diagnostics, fouling, and scaling, particularly in cases that require the separation of unwarranted solutes from solvents.
\end{abstract}

Published under license by AIP Publishing. https://doi.org/10.1063/5.0037120

\section{INTRODUCTION}

The study of crystallization of salts has been under the radar of the research community due to its numerous applications starting from salt manufacturing, pharmaceutical purification, seawater purification, cosmetic production, de-icing, and so on. ${ }^{1-4}$ Although several articles report about the precipitation mechanism and drying pattern of colloidal sessile droplets, ${ }^{5-7}$ much less attention has been given to the crystallization behavior of evaporating sessile droplets. The article by Kaya et al. deals with the effects of polyelectrolyte concentration of drops and the surrounding humidity on salt crystallization patterns such as concentric rings, and needle-like and chainlike structures are observed. ${ }^{8}$ Takhistov and Chang examined the crystal deposition of microliter droplets on substrates of different wettability. ' Concentric rings of salts were observed on hydrophilic surfaces; however, distinct crystals were produced on hydrophobic surfaces. ${ }^{9}$ Shin et al. reported a variety of three-dimensional crystalline deposits produced from droplets with high aspect ratio. ${ }^{10}$ The evaporation dynamics of saline drops suggest concentration and wettability influence the final precipitates. ${ }^{11}$ Exploring the mechanism behind diverse patterns due to droplet drying either by controlling or by suppressing the crystallization/efflorescence events may give further insights useful in fabricating miniaturized devices implemented as a diagnostic tool for bio-medical applications. $^{12-14}$ Fouling and scaling of salts causes damage to heat exchangers in industries, ${ }^{15}$ osmotic membranes, ${ }^{16}$ and microscale devices. ${ }^{17}$ Controlling crystallization is of prime importance in such applications. Another important aspect could be adopting prevention measures during the maintenance/repair of the buildings/structures (dam) and marine vessels (ships) exposed to the saline environment as repeated cycles of crystallization curtail their lifetime. 
During drying of a sessile drop (base fluid) containing other elements (nanoparticle, salt, etc.), the heterogeneous distribution of particle/solute molecules can be observed due to the droplet's internal flow behavior. The so-called "coffee ring" pattern emerges due to the capillary flow during evaporation, ${ }^{18}$ while another type of deposition is observed around the central region of the drop caused by Marangoni flow that arises from the surface tension gradient as a result of thermal or solutal gradient. ${ }^{19,20}$ Essentially, by manipulating the flow within an evaporating drop, one can determine the fate of the deposits on the substrate. Different researchers employed multiple techniques to control the flow behavior inside the drop such as by varying temperature gradients, ${ }^{21}$ changing substrate wettability, incorporation of surfactants, ${ }^{23}$ external acoustic stim$\mathrm{uli}^{24}$ and magnetic stirring; ${ }^{25}$ henceforth, the discussion is limited only to the effects of vapor-mediated interactions due to Marangoni convection.

Malinowski et al. demonstrated real-time control of the Marangoni flow within the evaporating droplet by introducing an ethanol vapor source. ${ }^{26}$ They had shown, by varying the source location, flows can be modulated spatially and temporally to generate surface patterns in a controlled manner. Kim et al. successfully punctured a dry hole within an aqueous liquid film (thickness greater than $100 \mu \mathrm{m})$ by exposing the film to an overhanging isopropyl alcohol drop. $^{27,28}$ This phenomenon was attributed to the vapor-mediated Marangoni effect, which promotes the rapid de-wetting of the liquid film. Recently, Kabi et al. reported that a single sessile water droplet could be separated into two distinct liquid films using vapormediated interactions because of the Marangoni flow induced by the differential adsorption of ethanol vapor generated from a pendant drop. $^{29}$ Cira et al. showed vapor-mediated motility of the binary mixture droplet due to Marangoni convection. ${ }^{30}$ Vapor-mediated interaction in droplets is also known to induce thermal Marangoni convection leading to attracting floating droplets. ${ }^{31}$ Sen et al. have also reported the transport of droplets using vapor-mediated interactions. $^{32}$ All these articles point out that the vapor-mediated interaction can be regarded as one of the means of realizing the contact line, which can be further explored in the fields of surface patterning and deposition dynamics.

Thereby, this article proposes a unique application of vapormediated interaction in the domain of crystal deposition. A gradient of surface tension due to the asymmetric vapor adsorption generates vigorous Marangoni flow. This can be leveraged to modulate the site of crystal deposition. Our experiments have demonstrated that introducing a non-uniform ethanol vapor field (through a pendant drop) around a sessile saline drop results in localized crystal precipitates. To the best of our knowledge, this phenomenon has never been reported. In this article, we focus on the interplay of physics in between parameters such as contact line dynamics, flow dynamics, and timescales that govern the location of crystal deposits. This study does not intend to delve into the fundamental behaviors of crystal arrangements and their transient growth dynamics from evaporating saline drops in the vapor-mediated surroundings. We first present the global observations of contact line dynamics and provide justified explanations by analyzing the flow physics. Appropriate scaling arguments reveal meaningful insights into the flow dynamics due to Marangoni convection, and a numerical solution of the concentration field of ethanol corroborates the experimental findings. Further experiments with different salt solutions ascertain the universal character of the proposed methodology responsible for such unique deposition dynamics of salt crystals.

\section{MATERIALS AND METHODS}

\section{A. Materials}

The analytical reagent sodium chloride $(\mathrm{NaCl})$ is procured from Rankem ${ }^{\circledR}$. Other salts such as copper(II) chloride dihydrate $\left(\mathrm{CuCl}_{2} .2 \mathrm{H}_{2} \mathrm{O}\right)$, ammonium sulfate $\left[\left(\mathrm{NH}_{4}\right)_{2} \mathrm{SO}_{4}\right]$, and magnesium sulfate heptahydrate $\left(\mathrm{MgSO}_{4} .7 \mathrm{H}_{2} \mathrm{O}\right)$ are commercially available from Merck. The aqueous solution is prepared by dissolving two different concentrations $(0.15 \mathrm{M}, 1 \mathrm{M})$ of salts in DI water. The properties of the salts are provided in Table I. Fluorescent polystyrene particles $(860 \mathrm{~nm})$ used for $\mu$-PIV are procured from Thermo Fisher Scientific.

\section{B. Experimental setup}

The schematic of the experimental setup is shown in Fig. 1. Optically flat plain glass slides procured from Blue Star(C) are cleaned by propan-2-ol (commonly known as isopropanol) followed by a de-ionized (DI) water rinse and blow-dry. $0.4 \pm 0.1 \mu \mathrm{l}$ of the saline $(\mathrm{NaCl})$ solution droplet is gently placed on the glass substrate using Thermo Scientific Finnpipette ${ }^{\circledR}$ (adjustable volume $0.2 \mu \mathrm{l}-2 \mu \mathrm{l}$, accuracy $\pm 12 \%$ for $0.2 \mu \mathrm{l}$ ). As the droplet evaporates, the contact line dynamics of the solvent droplet is observed from the top, using an optical microscope. The droplet is illuminated by a light source in-line with the objective lens of the microscope. The PCO camera (CMOS) attached to the microscope captures the reflected light. The top view images of the solvent droplet are captured at $2.14 \mathrm{fps}$ for its evaporation lifetime. Besides, a digital camera [D7200 Nikon fitted with a zoom lens assembly (Navitar)] is also used to capture

TABLE I. Properties of the different salts used in the experiments.

\begin{tabular}{lccc}
\hline \hline Salts & $\begin{array}{c}\text { Chemical } \\
\text { formula }\end{array}$ & $\begin{array}{c}\text { Molecular } \\
\text { weight }\end{array}$ & $\begin{array}{c}\text { Solubility in water } \\
\text { (g/l) }(293 \mathrm{~K})\end{array}$ \\
\hline Sodium chloride & $\mathrm{NaCl}$ & 58 & 360 \\
Copper chloride dihydrate & $\mathrm{CuCl}_{2} .2 \mathrm{H}_{2} \mathrm{O}$ & 170 & 76 \\
Ammonium sulfate & $\left(\mathrm{NH}_{4}\right)_{2} \mathrm{SO}_{4}$ & 132 & 75 \\
Magnesium sulfate heptahydrate & $\mathrm{MgSO}_{4} .7 \mathrm{H}_{2} \mathrm{O}$ & 246 & 35 \\
\hline \hline
\end{tabular}



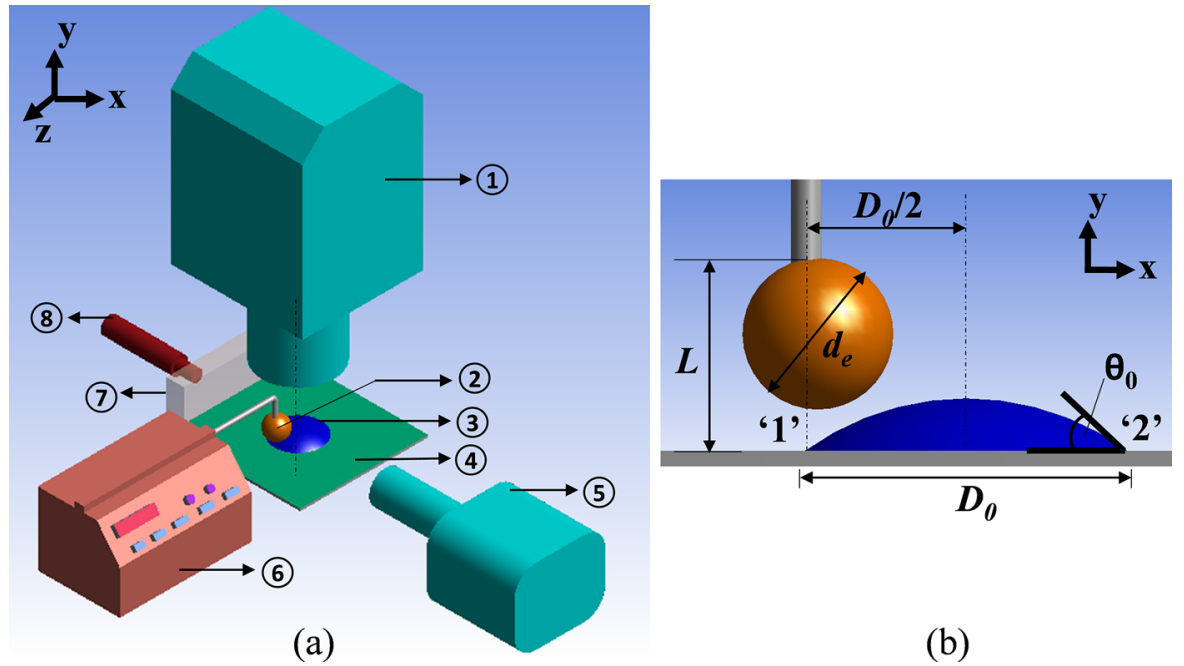

(a)

shadowgraph images from the side view [refer to Fig. 1(a)]. The LED light source from KARL STORZ NDTec (TECHNO LED NOVA 150) is placed behind the droplet for shadowgraph imaging, as shown in Fig. 1(a). A diffuser plate is used in front of the light source to produce a homogeneous distribution of light. The shadowgraph images from the side view are captured at $1 / 5 \mathrm{fps}$. Since the droplet radius $\sim 0.9 \mathrm{~mm}$ is less than the capillary length $\left(l_{c}\right)\left(l_{c}=\sqrt{\frac{\sigma}{\rho g}}\right.$ $\sim 2.74 \mathrm{~mm}, \sim 74 \mathrm{mN} / \mathrm{m},{ }^{33,34}$ Bond number $B o=\frac{\rho g D_{o}^{2}}{\sigma} \sim 0.132<1$, where $D_{0}$ is the initial saline droplet diameter), the droplet must initially assume a shape of a spherical cap. The initial contact angle of the deposited $\mathrm{NaCl}$ droplet on glass is observed to be $30 \pm 50$ for the experimental concentration range $(0.15 \mathrm{M}$ and $1 \mathrm{M}$ of salt). Sghaier et al. ${ }^{35}$ studied the influence of contact angle on the concentration of $\mathrm{NaCl}$, and the values of contact angles observed in our experiment are consistent for the given concentration $(0.15 \mathrm{M}$ and $1 \mathrm{M})$.

Initially, the evaporation dynamics is studied only for the saline drop, and it serves as a base case. Furthermore, the experiments are carried out to understand the effect of vapor-mediated interaction, as discussed previously. We introduce an ethanol vapor source near the solvent droplet. This is done by strategically positioning a pendant ethanol drop [volume of the ethanol drop $\left(V_{e}\right) \sim 2 \mu \mathrm{l}$ ] from a syringe pump near the solvent droplet, as shown in Fig. 1(b). The side at which the pendant ethanol droplet is positioned with respect to the solvent droplet is hereafter referred to as side " 1 " in the current literature. Subsequently, the other side is named side " 2 " [refer to Fig. 1(b)]. The pendant ethanol droplet and the solvent droplet are positioned in the same axial plane [ $x-y$ plane, as shown in Fig. 1(b)]. The tip of the needle of the ethanol droplet is placed at the height of $L \sim 2.3 \mathrm{~mm}$ from the substrate and is placed laterally at the edge of the solvent droplet. The motion control of the syringe pump and positioning of the droplet in the given configuration are done using high precision transitional stages from HOLMARC. Due to the high volatility of ethanol, the ethanol droplet shrinks very fast. However, the volume of the ethanol droplet is approximately maintained constant throughout each experimental run. This is achieved by pumping a constant flow rate $(1 \mu \mathrm{l} / \mathrm{min} \sim$ evaporation rate of ethanol at STP) of ethanol from the syringe pump, which replenishes the efflux of the vapor from the ethanol droplet due to evaporation. If the ethanol drop $\left(d_{e}\right)$ diameter grows bigger than the initial diameter, the pump is turned off. The pump is turned on as the ethanol droplet shrinks again. The shape of the droplet is spherical (diameter $\sim 1.56 \mathrm{~mm}$ ). The diameter of the needle $(D)$ is $0.45 \mathrm{~mm}$. The Worthington number Wo $=\frac{\rho_{e} g V_{e}}{\pi \sigma_{e} D} \sim 0.146<1$, where $\rho_{e}$ is the density of ethanol and $\sigma_{e}$ is the surface tension of ethanol, ensures the pendant ethanol droplet is spherical.

The evaporation dynamics are observed for two concentrations of $\mathrm{NaCl}$ solution: $0.15 \mathrm{M}$ (lower concentration) and $1 \mathrm{M}$ (higher concentration) solutions. The evaporation experiments are conducted for the solvent droplet without the presence of a pendant ethanol droplet (referred to as a lone solvent droplet in the manuscript) and for the solvent droplet placed near the pendant ethanol droplet (referred to as a solvent droplet with ethanol in the manuscript). Repeatability is ensured by conducting at least ten trials of every experiment in a controlled laboratory environment with an ambient temperature of 25 $\pm 2{ }^{\circ} \mathrm{C}$ and relative humidity of $45 \% \pm 5 \%$ (measured by TSP- 01 , Thorlabs).

\section{C. $\mu$-PIV for flow studies}

Micro-particle image velocimetry ( $\mu$-PIV) is done to obtain the qualitative and quantitative data of the flow field inside the solvent droplet. The images are captured (in the $z-x$ plane from the bottom of the solvent droplet) using an Imager Intense camera fitted to a Flowmaster MITAS microscope [field of view (FOV): 1000 $\times 600 \mu \mathrm{m}^{2}$, depth of field: $28 \mu \mathrm{m}$ ]. An Nd:Yag laser (NanoPIV, Litron Laser) is used for volumetric illumination of the droplet. Neutrally buoyant fluorescent monodispersed polystyrene particles of 860 $\pm 5 \mathrm{~nm}$ are added to the solvent as tracer beads. For a single sessile droplet (hydrophilic), a lower magnitude of the velocity of flow [of $\sim \mathrm{O}(10) \mu \mathrm{m} / \mathrm{s}$ ] within the droplet is expected. ${ }^{22}$ Hence, the images are acquired using a single frame-single pulse technique, and the images are captured at $1 \mathrm{fps}$. In the presence of ethanol vapor, a higher 
order of magnitude of the velocity of flow inside a sessile droplet [of $\sim \mathrm{O}(10) \mathrm{mm} / \mathrm{s}$ ] is expected ${ }^{36-38}$ due to Marangoni convection. Hence, the images are acquired using a double frame-double pulse technique. The image pairs are captured at $5 \mathrm{fps}$ with an intra-pair time delay of $8000 \mu \mathrm{s}$. It has been ensured that 3 pixels -4 pixels of particle shift is maintained for both cases mentioned. This is deemed optimal for PIV computation.

\section{RESULTS}

\section{A. Global observations of contact line (CL) dynamics}

The contact line (outer rim of the droplet) dynamics has been a topic of interest as it plays fundamental roles in various physicalchemical-biological-mechanical phenomena. ${ }^{39}$ The understanding of contact line (CL) dynamics remains important as the CL's pinning/depinning mechanism often determines the location of the deposition, after evaporation induced drying of multicomponent solutions. Further insights may bring out several unanswered questions about the crystallization dynamics of saline droplets.

The following are the observations from the experiments: In case of a saline drop undergoing evaporation $(0.15 \mathrm{M} \mathrm{NaCl})$ (up to $t / t_{f}<0.3$, where $t$ is the instantaneous time and $t_{f}$ is the total evaporation time of the solvent droplet), initially, the contact line is pinned on the surface. This may be due to slight surface heterogeneity. During the evaporation of solvent droplets with lower salt concentrations $(0.15 \mathrm{M} \mathrm{NaCl})$, the $\mathrm{CL}$ generally starts depinning at $t / t_{f}>0.4$ [see Fig. 2(a)] from a random edge, while there is a random edge that remains pinned on the initial CL. As per our observations (considering multiple runs), there is no preferential location for CL departures from any random direction of the perimeter. However, while a portion of the rim recedes inward (toward center), other parts remained pinned to the initial contact line, which means the CL is asymmetric [Fig. 2(a)]. The solvent droplet continues shrinking, and eventually, the crystals are formed near the pinned edge of the CL. Similar observations were also reported by Giri et al. . $^{4,41}$ However, Shahidzadeh et al. ${ }^{42}$ observed that the crystal precipitates are formed at the droplet's center. It is well known that a little change in the surface's micro-roughness may lead to contact line pinning. It also leads to the contact line and contact angle hysteresis. ${ }^{43}$ The difference in our results could be attributed to the same. ${ }^{44}$ We found the droplet remains pinned throughout its evaporation lifetime for a higher salt concentration $(1 \mathrm{M} \mathrm{NaCl})$ and later forms a ring of crystals, as shown in Fig. 2(c). Thus, the CL is dependent on the salt concentration.

However, when we introduce a pendant ethanol droplet over the solvent drop, depinning of the contact line of the solvent droplet always occurs from side "1" (near ethanol), irrespective of the salt (a)

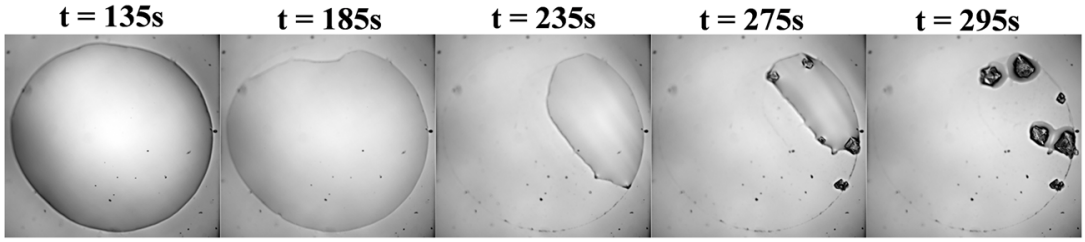

$\mathbf{t}=\mathbf{5 0 s}$

$\mathbf{t}=\mathbf{1 4 0 s}$

$\mathbf{t}=\mathbf{1 7 5 s}$

$\mathbf{t}=\mathbf{2 0 0 s}$

$\mathbf{t}=\mathbf{2 2 5 s}$

(b)

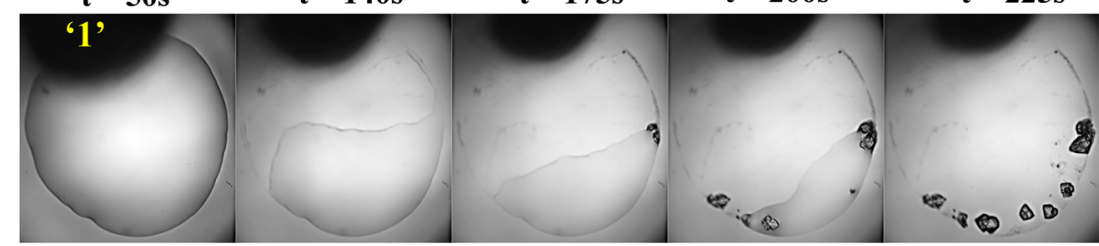

(c)

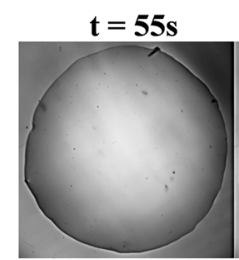

$\mathbf{t}=\mathbf{1 0 5 s}$

$\mathrm{t}=\mathbf{1 6 0 s}$

$t=195 s$
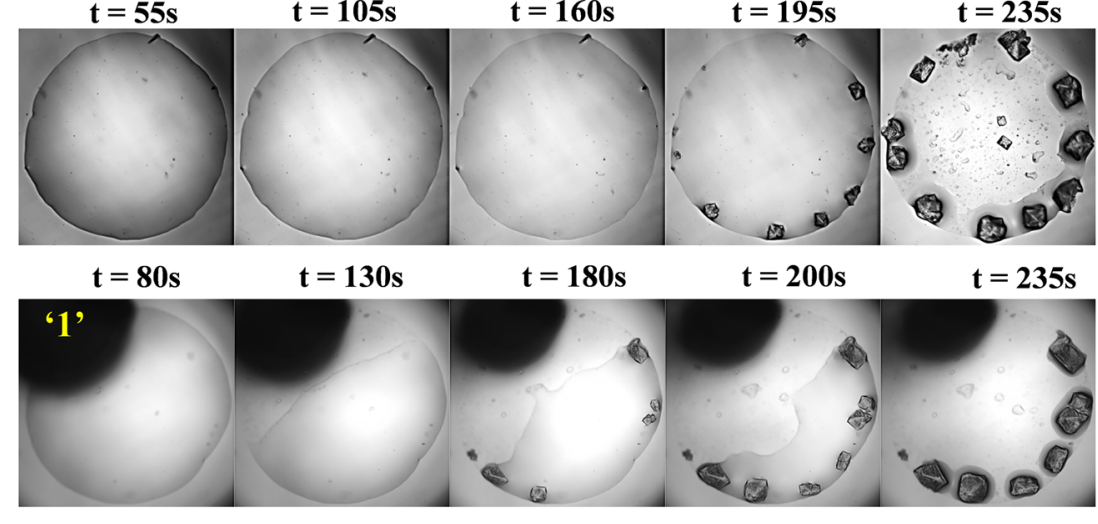

$\mathrm{t}=180 \mathrm{~s}$

$t=235 s$

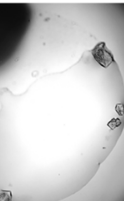

\begin{abstract}
$\mathbf{t}=200 \mathrm{~s}$
\end{abstract}
$\mathbf{t}$

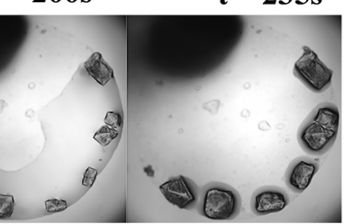

2

$0.7 \mathrm{~mm}$
FIG. 2. Global observations: contact line dynamics. Snapshots of images of a droplet are captured from the top at different time intervals under an optical microscope. The time instant at which the image is captured is given above the image. A series of images show the transient drying dynamics of a $0.5 \mu \mathrm{l}$ droplet on the glass substrate: (a) $0.15 \mathrm{M}$ $\mathrm{NaCl}$ solution, (b) $0.15 \mathrm{M} \mathrm{NaCl}$ solution in the presence of a pendant ethanol droplet hanging over it, (c) $1 \mathrm{M}$ $\mathrm{NaCl}$ solution, and (d) $1 \mathrm{M} \mathrm{NaCl}$ solution in the presence of a pendant ethanol droplet hanging over it. Marker "1" in images (b) and (d) represents the pendant ethanol droplet. Refer to Fig. 1 for the configuration of the pendant ethanol droplet. Multimedia view: https://doi.org/10.1063/5.0037120.1 
concentration [refer to Figs. 2(b) and 2(d)]. The novel observation from the experiments in ethanol's presence is that the CL of the solvent droplet always de-pins from the ethanol side (side " 1 "). This phenomenon remains invariant to the mole fraction of salt in the solvent droplet. Furthermore, as the solvent drop always de-pins from side " 1 " (in the presence of a pendant ethanol droplet), the final crystal precipitates are always obtained at side " 2 ." This has been observed consistently for many trials of experiments, and we present the multiple runs in the supplementary material, Fig. S1. To visualize the transient dynamics of the contact line from the top view, refer to the multimedia view (Fig. 2).

This entire phenomenon can be divided into different time regimes where we witness major events from placing the drop to its final drying (Fig. 2). The order is as follows: We observe depinning of the CL after a few seconds of deposition of the drop. Later, as the CL continues to de-pin, we see the inception of crystallization at some location. Finally, the entire solvent dries out, leaving only the solute crystals. As we are particularly interested in understanding flow physics leading to the preferred deposited pattern, among all the timescales, we further analyze the time regime from which we observe the first crystal $\left(t_{c r}\right)$ to the final precipitate $\left(t_{d r y}\right)$.

The time difference from the inception of the first crystal to the final precipitate $\left(t_{c r}-t_{d r y}\right)$ for a lone solvent droplet comes out low compared to that in corresponding cases where the ethanol drop is present (refer to Fig. 3); the timescale is normalized with $\left[\left(t_{c r}-t_{d r y}\right)_{1 \mathrm{M}}\right.$ of the $1 \mathrm{M}$ solution droplet in the presence of ethanol]. This signifies that even though crystals have started forming in some regions, a relatively higher volume of remnant liquid is still present in the case of drops that evaporate under the influence of the ethanol vapor field. On the contrary, the total time for evaporation is less for the solvent droplet $(0.15 \mathrm{M}$ concentration) in the presence of a pendant ethanol drop [see Fig. 4(a)] relative to the lone solvent droplet. To understand the rationale behind these observations, we further examine the flow inside the droplets with the help of $\mu$-PIV, discussed in Sec. IV A.
Figure 4 illustrates the temporal variation of different parameters such as the wetted contact area, contact diameter, and contact angle, the evaluation of which is important to figure out the characteristic behavior of the contact line. The parameters in Fig. 4 are non-dimensionalized for generalization as follows: The instantaneous wetted contact area $\mathrm{A}$ is divided by the initial wetted contact area $\mathrm{A}_{0}$. The instantaneous contact diameter $D$ is divided by the initial contact diameter $D_{0}$. The instantaneous contact angles $\theta_{1}$ and $\theta_{2}$ are divided by their respective initial contact angles $\theta_{01}$ and $\theta_{02}$. $\theta_{1}$ represents the contact angle of side " 1 " where the ethanol droplet is positioned, and $\theta_{2}$ corresponds to the other side. For the solvent droplet without the presence of an ethanol drop in the vicinity, the contact angle on one side is taken as $\theta_{1}$ and the other side as $\theta_{2}$. The time is non-dimensionalized with respect to the total time of evaporation of the solvent droplet " $\mathrm{t}_{0}$ " $(0.15 \mathrm{M})$ without the presence of ethanol. This is done because, among all four cases (low and high concentrations, each with and without ethanol), $t_{0}$ is the maximum time for evaporation. This approach of expressing each time in nondimensionalized form represents a clear picture of the differences in evaporation rates of all the cases. Every non-dimensional parameter plotted in Fig. 4 is an arithmetic average of the given parameter from four different trials at any given time instant. The corresponding error bars are the standard deviations of the four trials for each case.

The reduction of the wetted contact area is (at $t / t_{0} \sim 0.45$ ) gradual (slope $\sim 1$ ) as time progresses for the dilute solvent droplet $(0.15 \mathrm{M})$ in the absence of ethanol [Fig. 4(a)]. Depinning of the contact line due to evaporation has molecular origins. ${ }^{45}$ The CA decrease during evaporation creates unbalanced surface tension, due to which a force acts on the contact line. If the CL remains static, there is a pinning force exerted by the solid substrate (due to surface micro-roughness), which is more than the depinning force. When the depinning force dominates, the contact line shrinks. This balance of forces creates the stick-slip dynamics of the CL, making the contact area of the solvent droplet shrink gradually. The contact area (for a $0.15 \mathrm{M}$ droplet) shrinks steeply (slope $\sim 6.31$ ) at

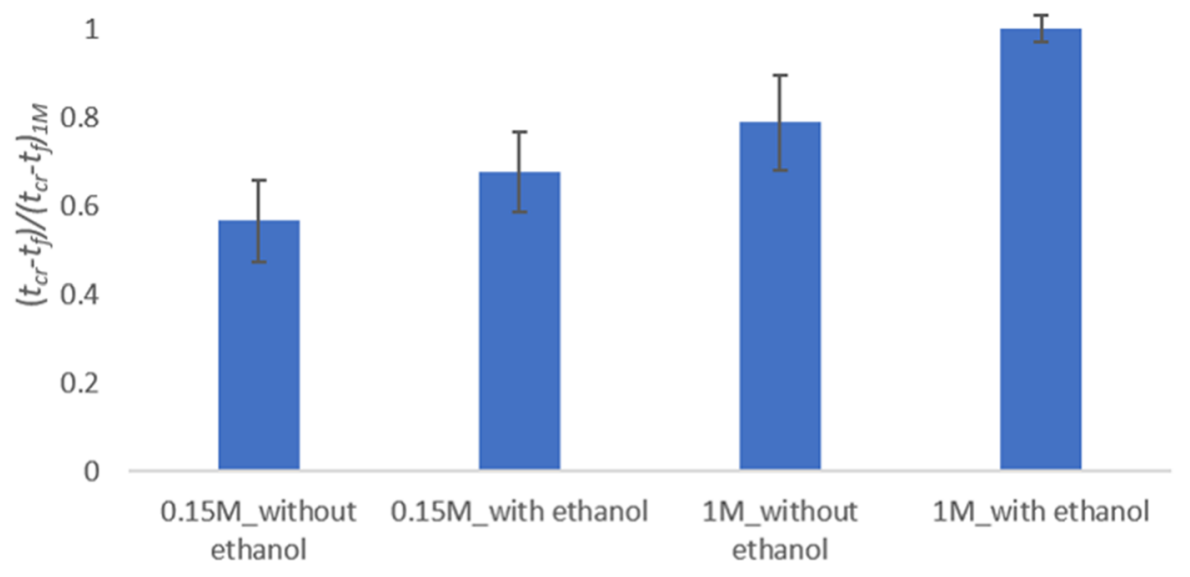

FIG. 3. Time elapsed from the inception of the first crystal to the complete dry out of the solvent droplet. Here, the $x$-axis represents the concentration of salt in the solvent droplet and the corresponding case. "0.15M_without ethanol" means the solvent droplet has a salt concentration of $0.15 \mathrm{M}$ and is placed on the substrate without the presence of ethanol in its vicinity. Correspondingly, "0.15M_with ethanol" means the pendant ethanol droplet is positioned adjacent to the solvent droplet, as shown in Fig. 1(b).

Experimental cases 

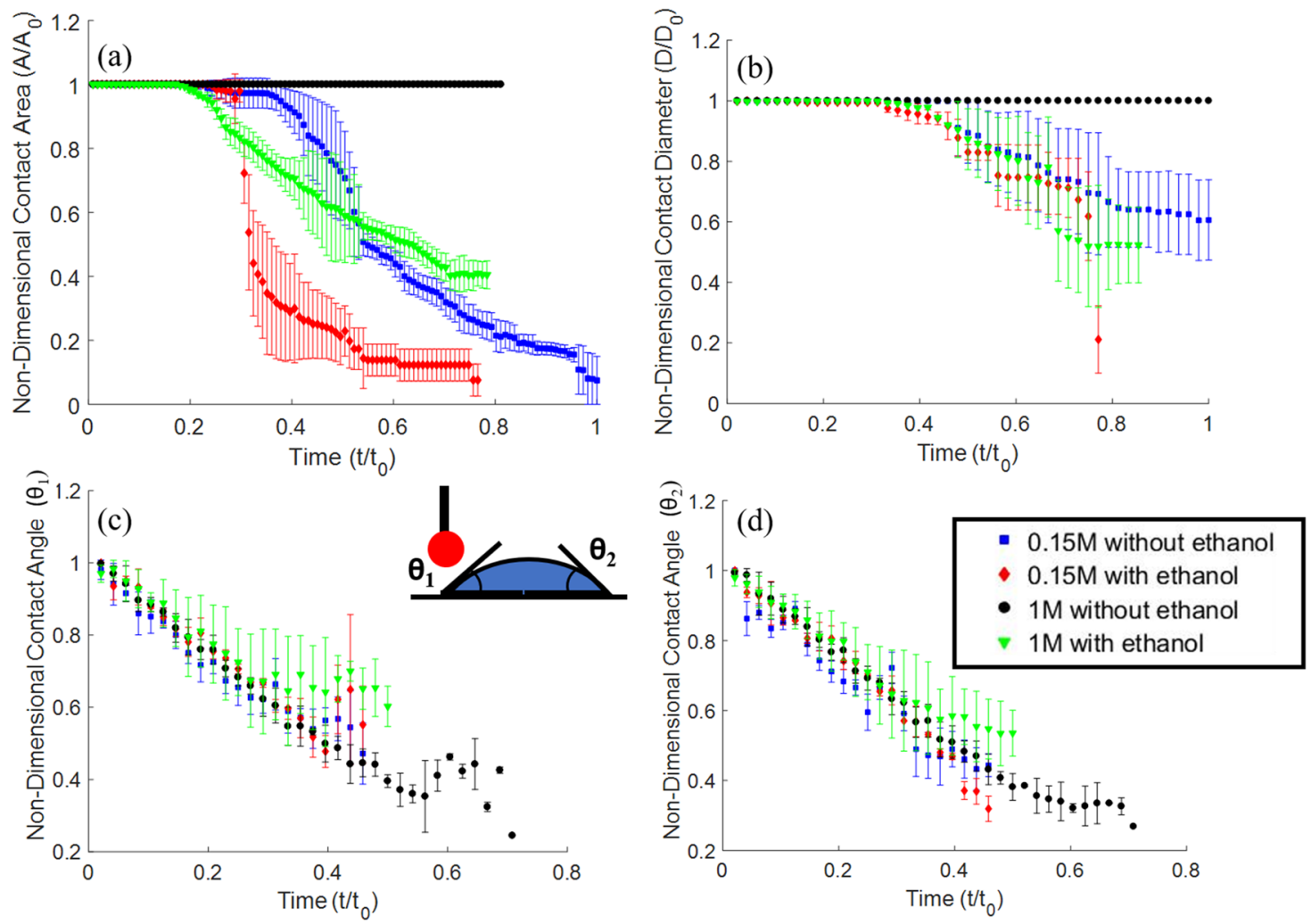

FIG. 4. Evolution of contact line dynamics. Temporal variation of the (a) contact area of the droplet, (b) contact diameter, (c) contact angle on side "1," i.e., the side of the ethanol droplet, and (d) contact angle on side "2." The legend adjacent to (d) is the same for (a), (b), and (c) in Fig. 4. All the variables are non-dimensionalized appropriately as discussed in Sec. III A. The error bars are the standard deviation taken from multiple (four) trials.

$\mathrm{t} / \mathrm{t}_{0} \sim 0.35$ in the presence of ethanol. The steeper contact area shrinkage occurs because of high Marangoni convection currents in the solvent droplet. The effects of Marangoni convection are explained in Sec. IV A. The droplets with small contact angles and heights can be approximated as thin liquid films on the surface. ${ }^{46}$ This is analogous to the formation of holes in a thin film when a pendant IPA vapor source was brought near the thin film. ${ }^{28}$ The hole of the thin film grows; ${ }^{28}$ the solvent droplet in our case shrinks in size due to the presence of a vapor source. The difference here is that the boundary is pushed from all directions of the hole formed on the thin film (because the vapor source is placed at the center of the hole), whereas the boundary of the solvent droplet is pushed from side " 1 " (because the vapor source is placed at the edge of the solvent droplet).

The contact area remains constant (slope $=0$ ) for a higher concentration $(1 \mathrm{M})$ solvent droplet as it remains pinned. Due to the higher solute concentration, the pinning force is always strong, which keeps the contact line pinned during the complete evaporation. Interestingly, the water-soluble crystals aggravate the pinning effect because of their hydrophilic nature. ${ }^{47}$ The increased wettability is one of the reasons for the pronounced pinning of droplets of $1 \mathrm{M}$ solution. However, the contact area shrinking for a higher concentration $(1 \mathrm{M})$ solvent droplet is observed in the presence of ethanol. The temporal decrease in the area is relatively smaller (slope $\sim 0.83$ ) than that in the lower concentration $(0.15 \mathrm{M})$ case in the presence of ethanol (slope $\sim 6.31$ ). However, its shrinkage despite the higher concentration of solute shows that an additional depinning force in the presence of ethanol vapor (apart from the depinning force due to evaporation) plays a decisive role in depinning of the droplet.

As noted from the side view of shadowgraph data, the contact diameter is plotted in Fig. 4(b). The stick-slip mode of the contact line is prominently observed for the cases where the reduction in the contact diameter is evident. Despite the sudden shrinkage in the wetted area [for a low concentration $(0.15 \mathrm{M})$ solvent droplet with ethanol case], the corresponding change in the contact diameter is not observed from the side view. This is because we can see the projected dimensions of spherical cap geometry from the side view. Once the contact area starts shrinking asymmetrically (as noted from Fig. 2), the contact diameter and contact angles would be different when seen from different directions of the side view. We see only the projected (normal) area from the side view, as explained earlier. We do not observe any significant difference in the contact angle of side " 1 " and side " 2 " of the solvent droplet due to the presence of ethanol. The contact angle measurement from the side view does not give any practical information when the droplet 
becomes spherically asymmetric, i.e., the contact angle measured from a different angle of side view would yield different values.

\section{B. Micro-particle image velocimetry ( $\mu$-PIV)}

To gain further insights into the internal hydrodynamics of the solvent drop, we have performed flow studies using $\mu$-PIV (Fig. 5). The settings used for $\mu$-PIV are already explained in Sec. II C. The post-processing of data is carried out using DAVIS software. All images are initially pre-processed by applying background subtraction. Post-processing is done by cross correlation of the double frame image pairs for a lone solvent droplet. Pairwise, cross correlation of sequential single frame images is done for the solvent droplet with ethanol. The interrogation window is maintained at $64 \times 64$ pixel $^{2}$ for the first pass and $32 \times 32$ pixel $^{2}$ for the subsequent passes with $50 \%$ overlap between two windows. The instantaneous vector fields, thus, obtained are temporally averaged (over the period pertaining to each regime) to obtain the final velocity vectors. The vectors shown in Fig. 5 correspond to the average flow velocity. The average velocity is calculated according to the equation $V_{\text {avg }}=\frac{1}{n} \sum_{i=1}^{n} V_{i}$, where $\mathrm{n}$ is the number of images over which the average is taken and $V_{i}$ is the velocity vector at a particular location.

First, we observe the flow inside the solvent droplet without ethanol as a base case [Fig. 5(b)]. The flow is observed in the planes $H / H_{0} \sim 0.1$ and $H / H_{0} \sim 0.7$, respectively [Fig. 5(b)]. The flow is observed to be radially inward in the plane $H / H_{0} \sim 0.1$ and radially outward in the plane $H / H_{0} \sim 0.7$. A similar flow was observed by Marin et al. ${ }^{20}$ and was attributed to Marangoni flow due to differential salt concentration. The flow eventually carries the molecules of $\mathrm{Na}^{+}$and $\mathrm{Cl}^{-}$to the edge of the droplet. As water evaporates, the salts crystallize at the edge due to supersaturation. The magnitude of velocity is observed to be $\sim O(10) \mu \mathrm{m} / \mathrm{s}$ [Fig. 5(b)]. Correspondingly, the Marangoni number due to the salt concentration difference is calculated as $\frac{U_{c s} L_{c}}{D_{(\text {Nacl-water })}}=\frac{10^{(-8)}}{10^{(-9)}} \sim O(10)$, where $U_{c s}$ is the characteristic Marangoni velocity scale due to the salt concentration gradient

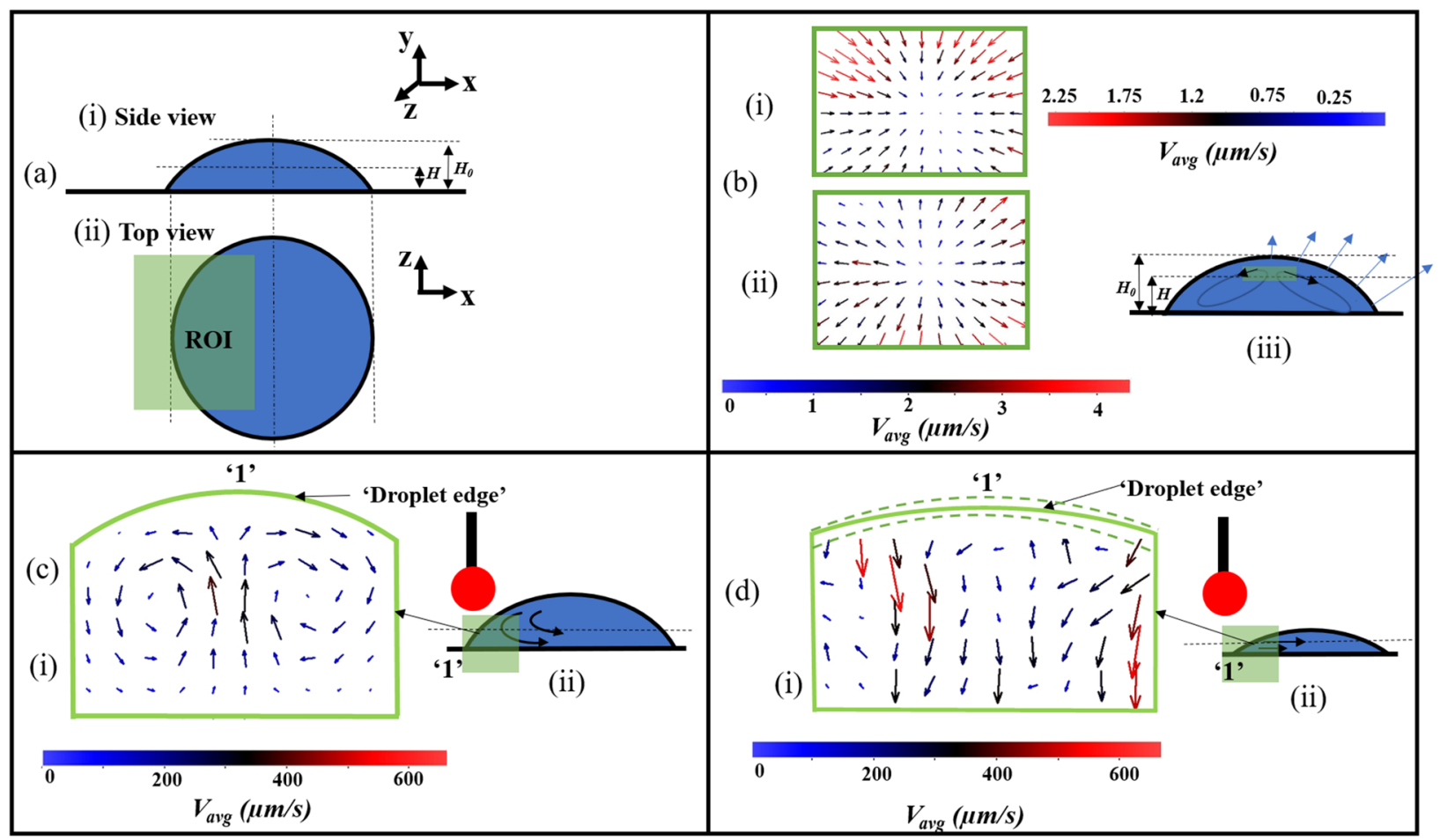

FIG. 5. (a) Schematic to represent the plane on which the video (multimedia view) is captured for the computation of $\mu$-PIV: (i) $\mu$-PIV is computed in a plane offset to the $x-z$ plane of the solvent droplet $(0.5 \mu \mathrm{l}$ droplet of $1 \mathrm{M}$ solution of $\mathrm{NaCl})$ at $H / H_{0}$, where $H$ is the distance of the given plane of measurement from the base and $H_{0}$ is the initial height of the solvent droplet; (ii) the curved surfaces represent the edge of the droplet. The squared boundary is the region of interest (ROI). (b) Vectors of the average velocity (averaged for a period of $100 \mathrm{~s}$ ) at the center of the solvent droplet without any droplet adjacent to it: (i) the vectors are measured in the plane $H / H_{0} \sim 0.1$, (ii) the vectors are measured in the plane $H / H_{0} \sim 0.7$, and (iii) schematic represents the solutal Marangoni flow in the solvent droplet. (c) (i) Vectors of the average velocity near the edge of the solvent droplet in the presence of the pendant ethanol droplet at side "1." (ii) Schematic representing uni-directional flow from side "1" of the droplet. The vectors averaged for a period of $50 \mathrm{~s}$ are plotted during the initial stages of evaporation. The contact line remains pinned during this time. (ii) Schematic representing circulatory flow observed near the edge of the droplet. (d) (i) Vectors of the average velocity near the edge of the solvent droplet in the presence of the pendant ethanol droplet at side "1." The vectors averaged for a period of $10 \mathrm{~s}$ are plotted during the final stages of evaporation when the contact line recedes. However, for the measurement period, the contact line does not recede much, and the dotted lines are representative of the same. In (c) and (d), the vectors of the average velocity in (i) correspond to the shaded region (green) in (ii) in the plane $H / H_{0} \sim 0.5$. Multimedia view: https://doi.org/10.1063/5.0037120.2 
as a result of differential evaporative flux across the surface of the saline droplet, $D_{(\mathrm{NaCl}-\text { water })}$ is the diffusion coefficient of $\mathrm{NaCl}$ in water $^{48}\left(\sim 10^{-9}\right)$, and $L_{c}$ is the characteristic length scale, which is the initial contact diameter of the droplet $D_{0}$.

When the pendant ethanol drop is placed near the solvent droplet, we observe a double toroidal flow in the $x-z$ plane of the solvent droplet. There is a bifurcation of flow near side " 1 " of the solvent droplet. The magnitude of flow velocity increases by $O\left(10^{2}\right)$ of magnitude. The Marangoni number due to the ethanol adsorption is calculated as $\frac{U_{c s} L_{c}}{D_{(\text {ethanol- water) }}}=\frac{10^{(-6)}}{10^{(-9)}} \sim O\left(10^{3}\right)$, where $U_{c s}$ is the characteristic Marangoni velocity scale due to adsorption of ethanol on the saline droplet, $D_{(\text {ethanol-water })}$ is the diffusion coefficient of ethanol in water ${ }^{49}\left(\sim 10^{-9}\right)$, and $L_{c}$ is the characteristic length scale, which is the initial contact diameter of the droplet $D_{0}$. A high Marangoni number is indicative of a robust convective flow in the droplet due to surface tension. The fluid at the edges of the droplet flows from side " 1 " to side " 2 ." To maintain continuity, the fluid at the center of the droplet moves from side " 2 " to side "1" [Fig. 5(c)]. Since the nature of the problem is symmetric on the $z-x$ plane [Fig. 5(a)], we see the expected symmetry in the form of a bi-toroidal motion of the fluid. Such fluid motion was observed with a single vapor source by Park et al. ${ }^{50}$ The Marangoni flow alters the solute deposition near the edge of the solvent droplet at side "1." The vigorous flow allows minimal ionic molecules to deposit at the pinned edge at side "1." The solute deposit at the other edge (far from the ethanol side) aggravates the surface heterogeneity. Hence, the pinning force increases with the increase in the solute concentration near the contact line, and vice versa. This makes the contact line susceptible to depinning on side " 1 " as the depinning force increases with evaporation. During later evaporation stages, the Marangoni flow transitions to a unidirectional flow from side " 1 " to side "2." Due to the continuous loss of fluids from side " 1 ," the contact line de-pins further [Fig. 5(d)]. Refer to the multimedia view (Fig. 5) for flow visualization.

Additional experiments are done with a pure water droplet and solvent droplet with low concentration $(0.15 \mathrm{M})$. The pure water droplet shows the capillary flow, and the saline droplet shows analogous findings observed in the case of the solvent droplet with high concentration (1M) (refer to the supplementary material, Fig. S2). This confirms that the vigorous flow observed in the presence of ethanol is not due to the presence of dissolved salt ions in the solvent droplet.

\section{DISCUSSIONS}

\section{A. Effect of flow on contact line dynamics and crystallization}

Contact line dynamics plays a determining role in finalizing the location of precipitates. More importantly, contact line dynamics is strongly influenced by the flow within the droplet, and vice versa. The pinned contact line results from surface heterogeneity and is aggravated by the solute molecules deposited by the solutal Marangoni flow because of the salt concentration gradient. As a result, the pinned contact line produces salt crystals everywhere on the edge of the droplet (1M concentration solvent droplet). If the solute concentration is low $(0.15 \mathrm{M})$, the contact line de-pins randomly from any side of the droplet and remains pinned at the other edge despite the salt deposition at the edge. The solutal Marangoni flow (due to salts) continues at the random pinned edge, and the salt crystallizes there.

In the presence of ethanol, the Marangoni flow dominates near side "1." 36 This leads to depinning of the droplet from side " 1 " as discussed in Sec. III B. The liquid volume is to be conserved, and the liquid is now confined to a smaller area. The distribution of liquid in this region might be uneven. The crystallization is initiated in the region where there is lesser liquid. Hence, although we see the early inception of the crystals, the time taken for the complete dry out further to inception is more in the "with ethanol" case (Fig. 3). We see the inception of the first crystals in the later stage of evaporation in "without ethanol" cases (as mentioned in Sec. III A), and the dry out time further to the inception of the first crystal is less because most of the liquid has been evaporated by that time.

The evaporation rate of the solvent droplet with a higher concentration $(1 \mathrm{M})$ was found to be more compared to that at the lower salt concentration (0.15M) [refer to Fig. 4(a)]. Jaiswal et al. observed an increase in the evaporation rate with the solute concentration, which was attributed to the chemical kinetics of hydration of ionic species in the polar fluid. ${ }^{51}$ Researchers have observed an increase in the evaporation rate with an increase in internal Marangoni convection. ${ }^{52}$ The increase in the evaporation rate of the $(0.15 \mathrm{M})$ solvent droplet with ethanol is attributed to the high convection induced by vapor-mediated interactions, as seen from $\mu$-PIV experiments.

\section{B. Marangoni flow due to vapor-mediated interactions}

We have ensured minimal external convection disturbances during the experiments conducted in a controlled laboratory condition. Furthermore, to confirm that the flow within the solvent drop is the consequence of the vapor induced interaction, we have performed computational simulations. Here, we have assumed diffusion to be the dominant mode of transport in the evaporation of droplets. The ethanol vapor is highly diffusive in air $\left(D_{\text {ethanol-air }}\right.$ $\sim 0.1149 \mathrm{~cm}^{2} / \mathrm{s}$ at STP). ${ }^{49}$ The solvent droplet evaporates slower than the pendant ethanol droplet, and hence, the solvent droplet size remains nearly constant for time of $t_{\text {diff }} \sim 10 \mathrm{~s}$. $t_{\text {diff }}$ is the approximate timescale for which the ethanol vapor field reaches the steadystate (refer to the supplementary material, Fig. 2). The size of the ethanol droplet $\left(d_{e}\right)$ remains nearly constant throughout the experiment. Based on the conditions mentioned above, it is logical to infer that the ethanol vapor field will reach a steady-state very fast. The exact problem formulation was first done by Epstein and Plesset. ${ }^{53}$ Hence, we solve the 3D (three-dimensional)steady-state diffusion equation $\nabla^{2} C=0$ to obtain the concentration field distribution/map of ethanol vapor around the saline drop using ANSYS FLUENT 2020 $\mathrm{R}^{1}$ (academic version) based on the Finite Volume Method (FVM). The geometry is made in Design Modeler, as shown in Fig. 1(b). The meshing is done in ANSYS with an element size of $0.3 \mathrm{~mm}$ for the larger domain (see the supplementary material, Fig. S3). Edge sizing of $0.1 \mathrm{~mm}$ is used on the surface of the droplet. The total number of elements is 321438 . The results change by less than $0.05 \%$, with the finer element size of $0.25 \mathrm{~mm}$ for the larger domain. Hence, the solution is confirmed to be grid-independent. 

lem:

The following boundary conditions are used to solve the prob-

(1) At the surface of the ethanol droplet, $C=C_{s}$, where $C_{s}$ is the saturation concentration of ethanol at STP.

(2) The flux of concentration at the surface of the glass and the surface of the solvent droplet is $\left(\frac{d C}{d n}\right)=0$.

(3) Since $C \propto 1 / r^{2}$ (where $r$ is the radial distance from the center of the ethanol droplet), if the domain edge is one order higher than the droplet dimension, we can consider it a far-field. The concentration of ethanol at far-field is $C=0$.

There is minuscule adsorption of ethanol into the solvent droplet. ${ }^{54}$ The concentration is non-dimensionalized by the saturation concentration of ethanol $\left(C_{s}\right)$; hence, the value of concentration ranges from 0 to 1 .

Figure 6 shows the ethanol concentration fields obtained from the simulations. The ethanol vapor engulfs the solvent droplet, as shown in Fig. 6(a). The asymmetric positioning of the ethanol droplet [Fig. 1(b)] results in a concentration gradient across the solvent droplet [Fig. 6(b)]. It is an established fact that the ethanol vapor in the vicinity positively adsorbs on the water droplet. ${ }^{55,56}$ Adsorption of ethanol occurs on the solvent droplet too. ${ }^{57}$ The asymmetric concentration field leads to the asymmetric adsorption of ethanol on the solvent droplet. It is thermodynamically proven that favorable adsorption leads to a decrease in surface tension. ${ }^{58}$ Local surface tension gradients drive the flow inside the solvent droplet. Considering a linear change in surface tension ${ }^{59}$ with concentration, $\Delta \sigma$ is estimated to be $\sim 0.1 \mathrm{mN} / \mathrm{m}$. The capillary number $C a=\frac{\mu U_{c}}{\Delta \sigma} \sim 10^{-2}$ indicates that the surface tension force dominates over viscous forces. Hence, we consider inertial scaling for the problem. Thus, the gradient of concentration indirectly reflects the inertial velocity due to
Marangoni stress on the surface of the droplet: $\frac{\Delta C}{L_{c}} \propto \frac{\Delta \sigma}{L_{c}} \propto \rho U_{c}{ }^{2}$, where $\Delta C$ is the concentration difference for the distance of the curve $L_{c}$ (characteristic length scale) on the surface of the solvent droplet, $\frac{\Delta C}{L_{c}}$ represents the surface gradient of concentration in the direction of the curve $L_{c}, \Delta \sigma$ is the corresponding difference in surface tension due to $\Delta C$, and $\rho U_{c}^{2}$ is the inertial scale, where $\rho$ is the density of the saline droplet and $U_{c}$ is the Marangoni velocity scale. The Marangoni flow tries to equilibrate the concentration gradient of ethanol. However, continuous adsorption from side " 1 " and desorption/evaporation of ethanol molecules from side " 2 " sustain the Marangoni flow.

With this theoretical basis, the direction of the flow inside the solvent droplet can be ascertained by knowing the direction of the maximum surface gradient of concentration. This has been quantified along four distinct imaginary curves [Fig. 6(c)] placed right on top of the outer plane of the spherical cap (sessile saline drop). For curves 1 and 2, the concentration values are computed from side " 1 " to side " 2 ," i.e., the high vapor concentration to the low concentration domain. The peak of concentration is found near the intersection of curve 1 and curve 4 [Fig. 6(d)]. The surface gradient of concentration along any curve in Fig. 6(d) is the slope of the corresponding curve in Fig. 6(c). The concentration gradient along curve 1 (from side " 1 ") until the peak concentration is reached is $\left(\frac{\Delta C}{L_{c}}\right)_{\text {Curve1 }} \sim \frac{0.0061}{0.348}=0.18 \mathrm{~mm}^{-1}$. The concentration gradient along curve 4 until the peak concentration is $\left(\frac{\Delta C}{L_{c}}\right)_{\text {Curve } 4} \sim \frac{0.1759}{0.621}$ $=0.28 \mathrm{~mm}^{-1}$. Here, the concentration difference $(\Delta C)$ is nondimensional, and the length is in millimeters. The concentration gradient is more along curve 4 . The concentration along curve 4 is symmetric. Therefore, at the intersection of curve 1 and curve 4 , there is a bifurcation of flow. The fluid is pulled along curve 4 symmetrically from the $x-y$ plane. The concentration continuously
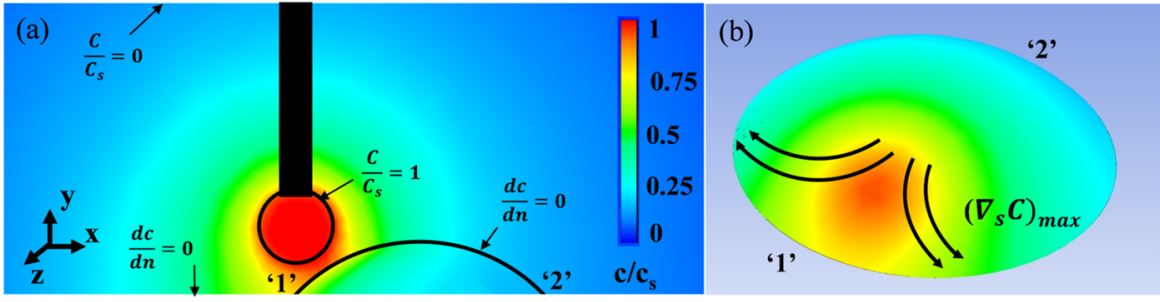

(c)

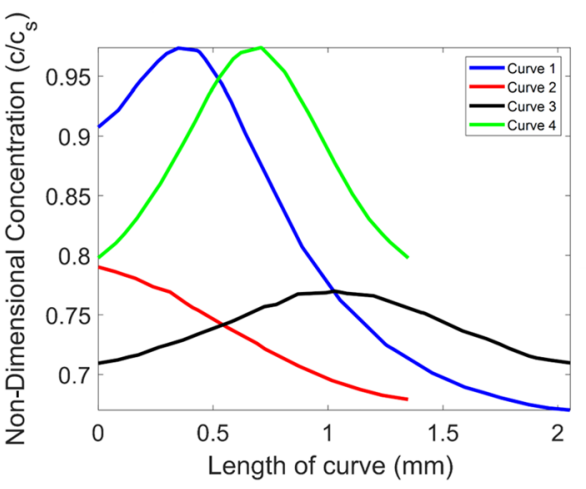

(d)

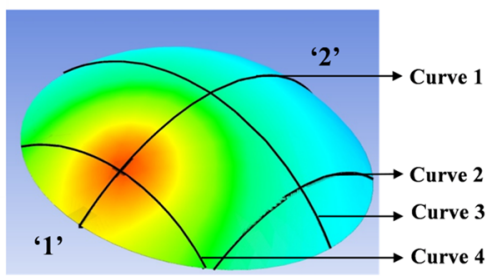

Phys. Fluids 33, 012101 (2021); doi: 10.1063/5.0037120
FIG. 6. Steady-state contours of concentration (a) of ethanol vapor around the solvent droplet in the $x-y$ cross-sectional plane passing through the center of the solvent droplet and (b) on the surface of the solvent droplet. The curved arrows point toward the maximum surface gradient of concentration $\left(\nabla_{s} C\right)_{\max }$, which are normal to the contours of concentration on the surface. (c) Variation of concentration along the length of different curves, as shown in (d). The curves are drawn on the surface of the solvent droplet. Curve 1 lies on the $x-y$ plane, and curve 2 is at an offset of $0.65 \mathrm{~mm}$ from the $x-y$ plane. The values of concentration plotted in (c) for curve 1 and curve 2 are from side " 1 " to side " 2. " Curves 3 and 4 lie on the $y-z$ plane and at $0.65 \mathrm{~mm}$ offset to the $y-z$ plane, respectively, and are symmetric. (d) Representation of curves. The data are plotted for the initial stages of evaporation of the solvent droplet $\left(t / t_{f} \sim 0.1-0.2\right)$ where the dimension of the solvent droplet is taken accordingly. 


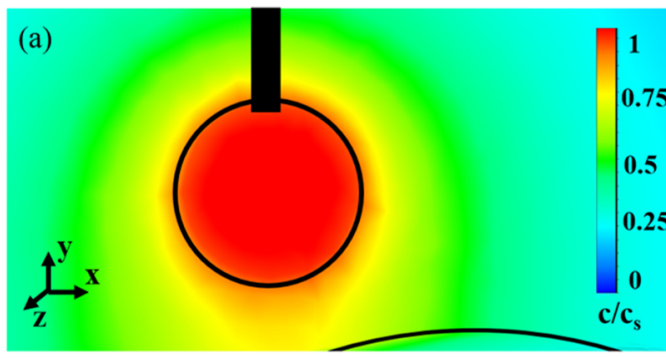

(c)

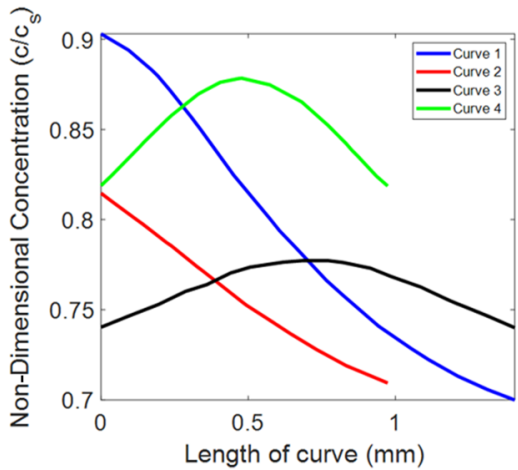

(b)

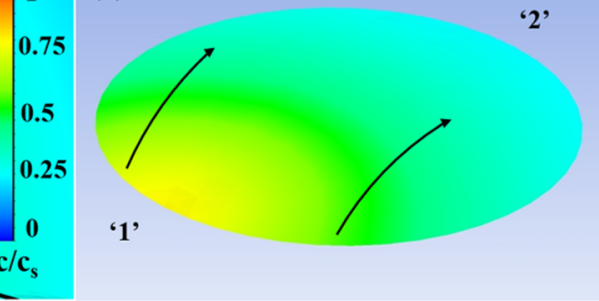

(d)

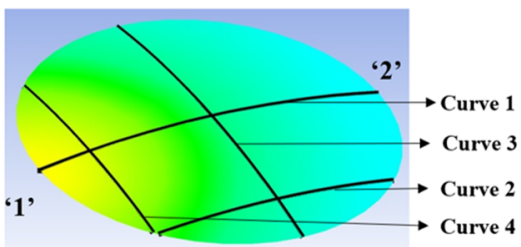

FIG. 7. Steady-state contours of concentration (a) of ethanol vapor around the solvent droplet in the $x-y$ cross-sectional plane passing through the center of the solvent droplet and (b) on the surface of the solvent droplet. The curved arrows point toward the maximum surface gradient of concentration $\left(\nabla_{s} C\right)_{\max }$, which are normal to the contours of concentration on the surface. (c) Variation of concentration along the length of different curves, as shown in (d). The curves are drawn on the surface of the solvent droplet. Curve 1 lies on the $x-y$ plane, and curve 2 is at the offset of $0.5 \mathrm{~mm}$ from the $x-y$ plane. Curves 3 and 4 lie on the $y-z$ plane and at $0.5 \mathrm{~mm}$ offset to the $y-z$ plane, respectively, and are symmetric. (d) Representation of curves. The data are plotted for the final stages of evaporation of the solvent droplet $\left(t / t_{f}\right.$ $\sim 0.5-0.7$ ), where the dimension of the solvent droplet becomes small with lower contact angles. decreases along curve 2 , and the surface tension builds up from side " 1 " to side "2" along curve 2 . This explains the double toroidal circulatory flow observed in $\mu$-PIV done on the $x-z$ plane [Fig. 5(c)]. The circulatory flow could cause an inhomogeneous distribution of salt (more at side "2" and less at side "1") in the saline droplet in the presence of ethanol vapor at side "1." Lowering the salt concentration at side " 1 " could lead to de-pinning from side " 1. "

There could be an additional reason for depinning of the droplet from side "1." This is due to the unidirectional flow in the saline droplet at later stages [Fig. 5(d)]. As the solvent droplet evaporates, the contact angle of the droplet reduces [Figs. 4(c) and 4(d)]. The changed shape of the solvent droplet changes the concentration of the ethanol vapor field around it. We assume a steady-state for this changed shape of the solvent droplet (the solvent droplet remains in the changed shape for a brief period) and solve for $\nabla^{2} C=0$ as done previously. Figure 7 shows the result of the steady-state concentration field for the changed shape of the solvent droplet.

The slope of curve 1 is $\left(\frac{\Delta C}{L_{c}}\right)_{\text {Curve1 }} \sim \frac{0.203}{1.405}=0.14 \mathrm{~mm}^{-1}$. The slope of curve 4 is $\left(\frac{\Delta C}{L_{c}}\right)_{\text {Curve } 4} \sim \frac{0.059}{0.468}=0.12 \mathrm{~mm}^{-1}$. The slope of curve 1 is higher, which indicates that the concentration gradient along curve 1 is also higher relative to that along curve 2. Therefore, the flow must be along curve 1 from side " 1 " to side " 2 ." The concentration continuously decreases from curve 2 , and the surface tension builds up from side " 1 " to side " 2 " along curve 2 . This explains the unidirectional flow in the $x-z$ plane as observed in $\mu$-PIV at the later (a1)

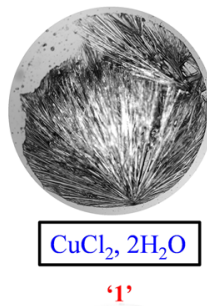

(a2)

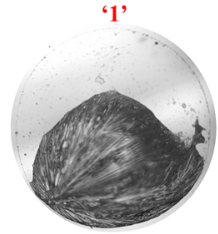

(b1)

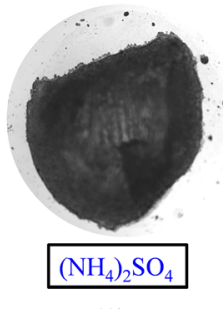

(b2)

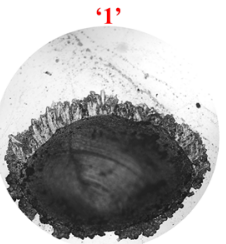

(c1)

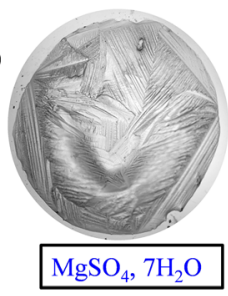

(c2)

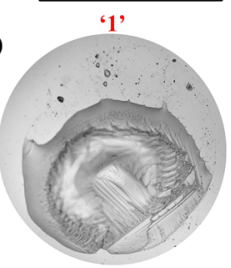

FIG. 8. Final precipitates of $1 \mathrm{M}$ aqueous solution of different salts: (a1) and (a2) $\mathrm{CuCl}_{2}, 2 \mathrm{H}_{2} \mathrm{O}$, (b1) and (b2) $\left(\mathrm{NH}_{4}\right)_{2} \mathrm{SO}_{4}$, and (c1) and (c2) $\mathrm{MgSO}_{4}, 7 \mathrm{H}_{2} \mathrm{O}$. The top row (a1), (b1), and (c1) corresponds to the without ethanol cases, while the bottom row (a2), (b2), and (c2) indicates the drying patterns in the presence of ethanol. Marker " 1 " in the bottom row shows the position of ethanol with respect to the drop. 
stages of evaporation of the solvent droplet in the presence of ethanol [Fig. 5(d)]. If the height of the droplet is small, the flow from side "1" to side " 2 " cannot revert. This ultimately leads to depinning of the drop. The absolute value of adsorption and the corresponding values of surface tension are difficult to determine and may require further experiments. By knowing the concentration field, we can predict the direction of the flow and explain depinning of the droplet successfully.

\section{UNIVERSALITY OF THE PHENOMENON}

Once we have established that by using vapor-mediated interaction, one can control the crystal precipitation site; we have further tested this principle for other salts $\left[\mathrm{CuCl}_{2}, 2 \mathrm{H}_{2} \mathrm{O}\right.$; $\left(\mathrm{NH}_{4}\right)_{2} \mathrm{SO}_{4}$; and $\left.\mathrm{MgSO}_{4}, 7 \mathrm{H}_{2} \mathrm{O}\right]$ to check the universal character of this phenomenon. Figure 8 illustrates the final precipitate images of these three salts, which exhibits similar results corresponding to the $\mathrm{NaCl}$ solvent droplet. The images confirm that solvent depletion has occurred from the ethanol side and the contact line has receded from side "1" (Fig. 8). Thereby, we observe all crystals accumulating at a location away from where the pendant ethanol drop was positioned (refer to Fig. 8, bottom row). This consolidates the claim of controlling the salt crystallization site attributed to the flow dynamics induced by the asymmetric vapor field around the solvent droplet. The results obtained from the experiments with other salts demonstrate the universality of the concept presented here. Whether the same phenomena can be observed irrespective of the choices of surfaces is, of course, a subject that needs further verification.

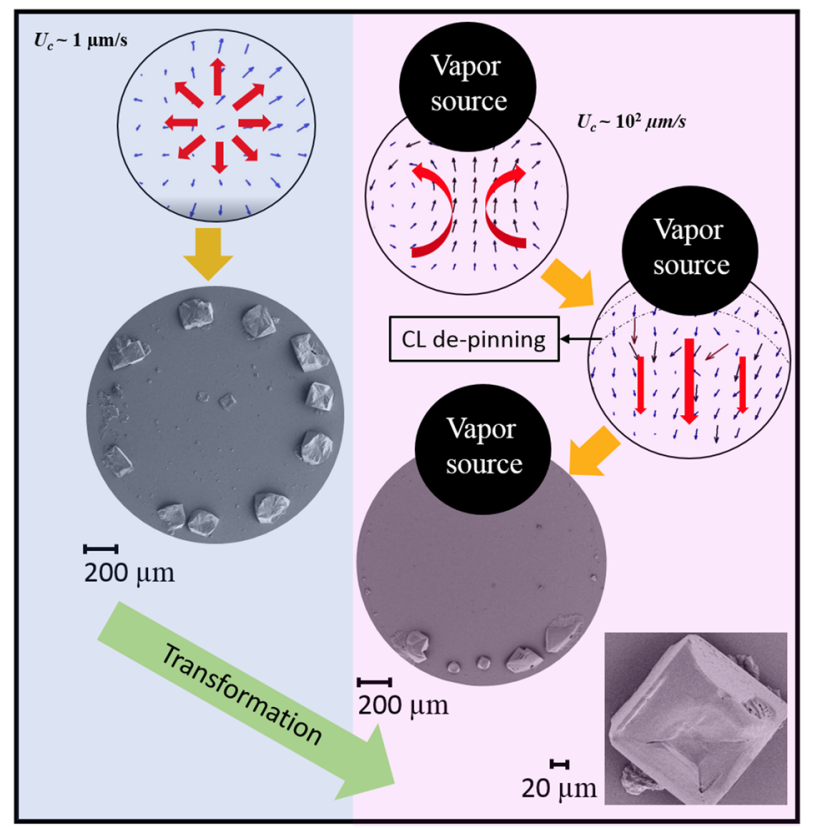

FIG. 9. Transformation of the salt deposit due to Marangoni advection induced by vapor-mediated interactions.

\section{CONCLUSIONS}

We summarize the major conclusions from the present work in Fig. 9. Researchers have shown that by controlling the flow, we can control the particle deposition. ${ }^{24,26,60-62}$ In this work, we have universally tailored the spatio-topological formation of crystals by controlling the flow, and the scattered distribution of precipitates is now limited to a concentrated region away from the vapor source. Contact line depinning and suppression of crystal deposits in the region were observed due to vapor-mediated interaction of droplets. The hypothesis is that the adsorption of ethanol vapor at the interfacial layer of the sessile saline drop subsequently locally changes its surface tension, generating Marangoni flow. This gives a satisfactory rationale to the flow behavior of the droplet. The methodology suggested in this article can be used as a probable tool to control crystal sites. Furthermore, this method can be potentially used in numerous fields ranging from water purification to pharmacy to portable diagnostics, particularly in cases requiring the separation of unwarranted solutes from solvents.

\section{SUPPLEMENTARY MATERIAL}

See the supplementary material for additional figures supporting the data.

\section{ACKNOWLEDGMENTS}

The authors acknowledge funding from the Ministry of Education, Government of India, and DRDO Chair Professorship.

The authors declare no competing financial interest.

\section{DATA AVAILABILITY}

The data that support the findings of this study are available within this article and its supplementary material. The chemical database for ethanol is openly available in Ref. 43.

\section{REFERENCES}

${ }^{1}$ M. J. Qazi, R. W. Liefferink, S. J. Schlegel, E. H. G. Backus, D. Bonn, and N. Shahidzadeh, "Influence of surfactants on sodium chloride crystallization in confinement," Langmuir 33, 4260 (2017).

${ }^{2}$ X. Wei, J. Yang, Z. Li, Y. Su, and D. Wang, "Comparison investigation of the effects of ionic surfactants on the crystallization behavior of calcium oxalate: From cationic to anionic surfactant," Colloids Surf., A 401, 107 (2012).

${ }^{3}$ M. Sammalkorpi, M. Karttunen, and M. Haataja, "Ionic surfactant aggregates in saline solutions: Sodium dodecyl sulfate (SDS) in the presence of excess sodium chloride $(\mathrm{NaCl})$ or calcium chloride $\left(\mathrm{CaCl}_{2}\right)$," J. Phys. Chem. B 113, 5863 (2009). ${ }^{4}$ J. Desarnaud, H. Derluyn, J. Carmeliet, D. Bonn, and N. Shahidzadeh, "Metastability limit for the nucleation of $\mathrm{NaCl}$ crystals in confinement," J. Phys. Chem. Lett. 5, 890 (2014).

${ }^{5} \mathrm{~N}$. Saxena, T. Naik, and S. Paria, "Organization of $\mathrm{SiO}_{2}$ and $\mathrm{TiO}_{2}$ nanoparticles into fractal patterns on glass surface for the generation of superhydrophilicity," J. Phys. Chem. C 121, 2428 (2017)

${ }^{6}$ H. Li, H. Luo, Z. Zhang, Y. Li, B. Xiong, C. Qiao, X. Cao, T. Wang, Y. He, and G. Jing, "Direct observation of nanoparticle multiple-ring pattern formation during droplet evaporation with dark-field microscopy," Phys. Chem. Chem. Phys. 18, 13018 (2016). 
${ }^{7}$ H. H. Lee, S. C. Fu, C. Y. Tso, and C. Y. H. Chao, "Study of residue patterns of aqueous nanofluid droplets with different particle sizes and concentrations on different substrates," Int. J. Heat Mass Transfer 105, 230 (2017).

${ }^{8}$ D. Kaya, V. A. Belyi, and M. Muthukumar, "Pattern formation in drying droplets of polyelectrolyte and salt," J. Chem. Phys. 133, 114905 (2010).

${ }^{9}$ P. Takhistov and H.-C. Chang, "Complex stain morphologies," Ind Eng. Chem. Res. 41, 6256 (2002).

${ }^{10}$ B. Shin, M.-W. Moon, and H.-Y. Kim, "Rings, igloos, and pebbles of salt formed by drying saline drops," Langmuir 30, 12837 (2014).

${ }^{11} \mathrm{X}$. Zhong, J. Ren, and F. Duan, "Wettability effect on evaporation dynamics and crystalline patterns of sessile saline droplets," J. Phys. Chem. B 121, 7924 (2017).

${ }^{12}$ A. V. Lyushnin, A. A. Golovin, and L. M. Pismen, "Fingering instability of thin evaporating liquid films," Phys. Rev. E 65, 021602 (2002).

${ }^{13}$ C. Ohm, M. Morys, F. R. Forst, L. Braun, A. Eremin, C. Serra, R. Stannarius, and R. Zentel, "Preparation of actuating fibres of oriented main-chain liquid crystalline elastomers by a wetspinning process," Soft Matter 7, 3730 (2011).

${ }^{14}$ D. Baranov, A. Fiore, M. Van Huis, C. Giannini, A. Falqui, U. Lafont, H. Zandbergen, M. Zanella, R. Cingolani, and L. Manna, "Assembly of colloidal semiconductor nanorods in solution by depletion attraction," Nano Lett. 10, 743 (2010).

${ }^{15} \mathrm{H}$. Müller-Steinhagen, "Cooling-water fouling in heat exchangers," Adv. Heat Transfer 33, 415 (1999).

${ }^{16}$ D. Vogel, A. Simon, A. A. Alturki, B. Bilitewski, W. E. Price, and L. D. Nghiem, "Effects of fouling and scaling on the retention of trace organic contaminants by a nanofiltration membrane: The role of cake-enhanced concentration polarisation," Sep. Purif. Technol. 73, 256 (2010).

${ }^{17}$ M. Schoenitz, L. Grundemann, W. Augustin, and S. Scholl, "Fouling in microstructured devices: A review," Chem. Commun. 51, 8213 (2015).

${ }^{18}$ K. Sefiane, "Patterns from drying drops," Adv. Colloid Interface Sci. 206, 372 (2014).

${ }^{19} \mathrm{H}$. Hu and R. G. Larson, "Marangoni effect reverses coffee-ring depositions," Phys. Chem. B 110, 7090 (2006).

${ }^{20}$ A. Marin, S. Karpitschka, D. Noguera-Marín, M. A. Cabrerizo-Vílchez, M. Rossi, C. J. Kähler, and M. A. Rodríguez Valverde, "Solutal Marangoni flow as the cause of ring stains from drying salty colloidal drops," Phys. Rev. Fluids $\mathbf{4}$, 041601 (2019)

${ }^{21}$ L. Bansal, A. Miglani, and S. Basu, "Morphological transitions and buckling characteristics in a nanoparticle-laden sessile droplet resting on a heated hydrophobic substrate," Phys. Rev. E 93, 042605 (2016).

${ }^{22}$ S. Dash, A. Chandramohan, J. A. Weibel, and S. V. Garimella, "Buoyancyinduced on-the-spot mixing in droplets evaporating on nonwetting surfaces," Phys. Rev. E 90, 062407 (2014).

${ }^{23}$ W. Sempels, R. De Dier, H. Mizuno, J. Hofkens, and J. Vermant, "Autoproduction of biosurfactants reverses the coffee ring effect in a bacterial system," Nat. Commun. 4, 1757 (2013).

${ }^{24}$ A. Sanyal and S. Basu, "Evolution of internal flows in mechanically oscillating sessile droplets undergoing evaporation," Chem. Eng. Sci. 163, 179 (2017).

${ }^{25}$ A. Rida and M. A. M. Gijs, "Manipulation of self-assembled structures of magnetic beads for microfluidic mixing and assaying," Anal. Chem. 76, 6239 (2004).

${ }^{26}$ R. Malinowski, G. Volpe, I. P. Parkin, and G. Volpe, "Dynamic control of particle deposition in evaporating droplets by an external point source of vapor," J. Phys. Chem. Lett. 9, 659 (2018).

${ }^{27}$ S. Kim, J. Kim, and H.-Y. Kim, "Dewetting of liquid film via vapour-mediated Marangoni effect,” J. Fluid Mech. 872, 100 (2019).

${ }^{28}$ S. Kim, J. Kim, and H.-Y. Kim, "Formation, growth, and saturation of dry holes in thick liquid films under vapor-mediated Marangoni effect," Phys. Fluids 31, 112105 (2019).

${ }^{29}$ P. Kabi, R. Pal, and S. Basu, "Moses effect: Splitting a sessile droplet using a vapor-mediated Marangoni effect leading to designer surface patterns," Langmuir 36, 1279 (2020).

${ }^{30}$ N. J. Cira, A. Benusiglio, and M. Prakash, "Vapour-mediated sensing and motility in two-component droplets," Nature 519, 446 (2015).
${ }^{31}$ D. Liu and T. Tran, "Vapor-induced attraction of floating droplets," J. Phys. Chem. Lett. 9, 4771 (2018).

${ }^{32}$ B. Majhy and A. K. Sen, "Evaporation-induced transport of a pure aqueous droplet by an aqueous mixture droplet," Phys. Fluids 32, 032003 (2020).

${ }^{33}$ B. R. Bzdek, R. M. Power, S. H. Simpson, J. P. Reid, and C. P. Royall, "Precise, contactless measurements of the surface tension of picolitre aerosol droplets," Chem. Sci. 7, 274 (2016).

${ }^{34} \mathrm{G}$. Jones and W. A. Ray, "The surface tension of solutions of electrolytes as a function of the concentration. I. A differential method for measuring relative surface tension,” Am. Chem. Soc. 59, 187 (1937).

${ }^{35} \mathrm{~N}$. Sghaier, M. Prat, and S. Ben Nasrallah, "On the influence of sodium chloride concentration on equilibrium contact angle," Chem. Eng. J. 122, 47 (2006).

${ }^{36}$ O. Hegde, S. Chakraborty, P. Kabi, and S. Basu, "Vapor mediated control of microscale flow in sessile droplets," Phys. Fluids 30, 122103 (2018).

${ }^{37}$ O. Hegde, P. Kabi, and S. Basu, "Enhancement of mixing in a viscous, nonvolatile droplet using a contact-free vapor-mediated interaction," Phys. Chem. Chem. Phys. 22, 014570 (2020).

${ }^{38}$ J. Park, J. Ryu, H. J. Sung, and H. Kim, "Control of solutal Marangoni-driven vortical flows and enhancement of mixing efficiency," J. Colloid Interface Sci. 561, 408 (2019)

${ }^{39}$ Y. Zhao, "Moving contact line problem: Advances and perspectives," Theor. Appl. Mech. Lett. 4, 034002 (2014).

${ }^{40}$ A. Giri, M. Dutta Choudhury, T. Dutta, and S. Tarafdar, "Multifractal growth of crystalline $\mathrm{NaCl}$ aggregates in a gelatin medium," Cryst. Growth Des. 13, 341 (2013).

${ }^{41}$ M. D. Choudhury, T. Dutta, and S. Tarafdar, "Pattern formation in droplets of starch gels containing $\mathrm{NaCl}$ dried on different surfaces," Colloids Surf., A 432, 110 (2013).

${ }^{42}$ N. Shahidzadeh, M. F. L. Schut, J. Desarnaud, M. Prat, and D. Bonn, "Salt stains from evaporating droplets," Sci. Rep. 5, 10335 (2015).

${ }^{43}$ P.-G. de Gennes, F. Brochard-Wyart, D. Quéré, P.-G. de Gennes, F. BrochardWyart, and D. Quéré, Capillarity Wetting Phenom (Springer New York, 2004), pp. 33-67.

${ }^{44} \mathrm{C}$. A. Wardand K. Sefiane, "Adsorption at the solidâĂşliquid interface as the source of contact angle dependence on the curvature of the three-phase line," Adv. Colloid Interface Sci. 161, 171 (2010).

${ }^{45}$ F. C. Wang and H. A. Wu, "Molecular origin of contact line stick-slip motion during droplet evaporation,” Sci. Rep. 5, 17521 (2015).

${ }^{46} \mathrm{M}$. Ikegawa and H. Azuma, "Droplet behaviors on substrates in thin-film formation using ink-jet printing," JSME Int. J., Ser. B 47, 490 (2005).

${ }^{47}$ S. A. McBride, R. Skye, and K. K. Varanasi, "Differences between colloidal and crystalline evaporative deposits," Langmuir 36, 11732 (2020).

${ }^{48} \mathrm{~V}$. Vitagliano and P. A. Lyons, "Diffusion coefficients for aqueous solutions of sodium chloride and barium chloride," J. Am. Chem. Soc. 78, 1549 (1956).

${ }^{49}$ See https://www.gsi-net.com/en/publications/gsi-chemical-database/single/259 -ethanol.html for the information about diffusion coefficient of ethanol.

${ }^{50}$ J. Park, J. Ryu, H. J. Sung, and H. Kim, "Control of solutal Marangoni-driven vortical flows and enhancement of mixing efficiency," J. Colloid Interface Sci. 561, 408 (2020).

${ }^{51}$ V. Jaiswal, A. R. Harikrishnan, G. Khurana, and P. Dhar, "Ionic solubility and solutal advection governed augmented evaporation kinetics of salt solution pendant droplets," Phys. Fluids 30, 012113 (2018).

${ }^{52}$ A. Chattopadhyay, R. K. Dwivedi, A. R. Harikrishnan, and P. Dhar, "Ferroadvection aided evaporation kinetics of ferrofluid droplets in magnetic field ambience," Phys. Fluids 32, 082001 (2020).

${ }^{53}$ P. S. Epstein and M. S. Plesset, "On the stability of gas bubbles in liquid-gas solutions," J. Chem. Phys. 18, 1505 (1950).

${ }^{54}$ O. Hegde, P. Kabi, S. Agarwal, and S. Basu, "Controlling self-assembly and buckling in nano fluid droplets through vapour mediated interaction of adjacent droplets,” J. Colloid Interface Sci. 541, 348 (2019).

${ }^{55} \mathrm{M}$. A. Wilson and A. Pohorille, "Adsorption and solvation of ethanol at the water liquid-vapor interface: A molecular dynamics study,” J. Phys. Chem. B 101, 3130 (1997). 
${ }^{56}$ P. Davidovits, C. E. Kolb, L. R. Williams, J. T. Jayne, and D. R. Worsnop, "Mass accommodation and chemical reactions at gas-liquid interfaces," Chem. Rev. 106, 1323 (2006).

${ }^{57}$ K. Sahu, V. F. McNeill, and K. B. Eisenthal, "Effect of salt on the adsorption affinity of an aromatic carbonyl molecule to the air-aqueous interface: Insight for aqueous environmental interfaces," J. Phys. Chem. C 114, 18258 (2010).

${ }^{58}$ A. A. Nepomnyashchy, M. G. Velarde, and P. Colinet, Interfacial Phenomena and Convection (Chapman and Hall/CRC, 2001).
${ }^{59}$ G. Vázquez, E. Alvarez, and J. M. Navaza, "Surface tension of alcohol water + water from 20 to 50.degree.C,” J. Chem. Eng. Data 40, 611 (1995).

${ }^{60}$ W. D. Ristenpart, P. G. Kim, C. Domingues, J. Wan, and H. A. Stone, "Influence of substrate conductivity on circulation reversal in evaporating drops," Phys. Rev. Lett. 99, 234502 (2007)

${ }^{61}$ Y. Zhang, S. Yang, and L. Chen, and J. R. G. Evans, "Shape changes during the drying of droplets of suspensions," Langmuir 24, 3752 (2008).

${ }^{62}$ L. Bansal, P. Seth, B. Murugappan, and S. Basu, "Suppression of coffee ring: (Particle) size matters," Appl. Phys. Lett. 112, 211605 (2018). 